\title{
MODELLING THE UNSTEADY DYNAMICS OF A TURBINE RESEARCH FACILITY
}

\author{
John P. Longley \\ Whittle Laboratory \\ University of Cambridge \\ 1 J.J. Thomson Avenue \\ Cambridge CB3 ODY, UK
}

\section{ABSTRACT}

The accuracy with which experimental investigations of turbine performance need to be undertaken require either a semi- or fully-automated control of the operating point as any variation can compromise the reliability of the measurements. Fundamentally, both the mass flow rate through the turbine and the applied brake torque need to be adjusted in real-time so that the required operating point is maintained.

This paper describes the development of a time accurate computational simulation of the unsteady dynamics of a largescale, low-speed turbine facility when its operating point is determined by a full-authority control system. The motivation for the development of the computational simulation was to be able to safely undertake parametric studies to refine the control system and to investigate the cause of monotonic excursions of the operating point which were observed after a major rebuild.

The monotonic excursions of the turbine operating point could only be reproduced by the computational simulation after an unsteady aerodynamic coupling between the turbine exit flow and the downstream centrifugal fan had been incorporated. Based on this observation a honeycomb was installed upstream of the fan in the turbine facility. This eliminated the monotonic excursions and the fractional noise of the operating point was reduced by $37 \%$. When combined with an earlier refinement of the control system the factional noise was reduced by a factor of three. This enables the number of repeated measurements to be reduced by nine and still obtain the same quality of data.

\section{BACKGROUND}

Turbine research facilities are used extensively in the investigation of fundamental performance and to determine the effects of novel geometries. The reliability of such investigations depends critically on the facility being at the correct operating point throughout the measurements. There is a wide variation in the design and operation of turbine facilities because they were each developed to investigate different aspects of aerodynamic and aerothermal performance.
There is little published data on the consistency with which turbine facilities maintain the operating point and what data is available is presented in a form appropriate to the specific investigations undertaken. A selection of operating point consistency information is shown in Table 1 for both transient and nominally steady-state turbine research facilities (the Peregrine turbine is discussed in this paper). For comparison, the value for a capacity rig is also included. The consistency values are good (less than one percent) and it is worth noting that steady-state facilities allow longer run times to acquire data which can then be averaged to improve consistency.

Table 1: Consistency of operating point for different types of turbine facilities.

\begin{tabular}{|l|c|c|}
\hline Type & Quantity & Consistency \\
\hline $\begin{array}{l}\text { Steady, manual control, } \\
\text { 1-stage, low-speed [1] }\end{array}$ & $\phi$ & $\pm 0.5 \%$ \\
\hline $\begin{array}{l}\text { Transient transonic } \\
\text { single stage [2] }\end{array}$ & shaft speed & $\pm 0.26 \%$ (typical) \\
\hline $\begin{array}{l}\text { Blowdown capacity rig } \\
\text { (no moving parts) [3] }\end{array}$ & $\dot{m} \sqrt{T_{t}} / p_{t}$ & $\begin{array}{c} \pm 0.02 \% \\
(95 \% \text { confidence) }\end{array}$ \\
\hline $\begin{array}{l}\text { Steady, closed loop, } \\
1.5 \text { stage low-speed [4] }\end{array}$ & $\phi$ & $(\sigma / \mu)_{120 \mathrm{~s}}=0.015 \%$ \\
\hline $\begin{array}{l}\text { Peregrine turbine } \\
\text { before refinement }\end{array}$ & $\phi$ & $(\sigma / \mu)_{120 \mathrm{~s}} \sim 0.015 \%$ \\
\hline $\begin{array}{l}\text { Peregrine turbine } \\
\text { after refinement [5] }\end{array}$ & $\phi$ & $(\sigma / \mu)_{120 \mathrm{~s}}=0.004 \%$ \\
\hline
\end{tabular}

For nominally steady-state turbine facilities there are two aspects to maintaining the correct operating point. Firstly, overcoming the effects of long term drift due to gradual changes in the operating conditions. Secondly, removing the effects of short timescale variations which are a combination of the unsteady dynamics of the facility and random unsteadiness. In addition, facilities which have an atmospheric inlet are also subjected to uncontrolled variations of the ambient conditions 
which occur over both the long timescale (diurnal and seasonal) and the short timescale (e.g. atmospheric turbulence). Closedloop turbine facilities are less susceptible to ambient conditions.

The effects of short timescale unsteadiness are often reduced by averaging repeated measurements over a period of time which is usually determined by experience and/or statistical analysis (e.g. by examining an autocorrelation). The disadvantage of repeated measurements is that it increases the duration, and therefore cost, of an experimental investigation. Also, averaging over a large number of data points can be adversely affected by long timescale variations. Further, any averaging process can only reduce the standard deviation of the short timescale unsteadiness if the data are independent.

Some of the effects of long timescale variations can be reduced during operation by adjusting the control parameters of the facility. Also by selecting the order in which repeated measurements are taken it may be possible to average-out any small linear variations of the operating point with time. For long timescale variations which occur between runs, e.g. seasonal ambient conditions, which cannot be directly eliminated by choice of the facility operating point, Evans \& Longley [5, 6] developed the Accounting Methodology (where multiple runs are best-fit correlated to a common datum).

\section{INTRODUCTION}

The Peregrine low-speed turbine facility at the Whittle laboratory has an atmospheric inlet, uses a centrifugal fan to provide the pressure drop across the turbine and an eddycurrent brake to absorb the output power. The rotational speed of the fan and the current applied to the brake must both be controlled to maintain the required flow coefficient and Reynolds number. A real time, full authority control system developed by Thomas [7] measures the ambient conditions along with the turbine operating point and adjusts the brake current and fan speed appropriately.

During the re-commissioning of the facility, after a major rebuild, a refinement to the control system (concerned with the PID control of the fan speed) reduced the factional noise in the turbine operating point by approximately 50\%, see Table 2. This gave a substantial improvement to the measurement quality but it was then observed that the operating point would, for several seconds, undergo a monotonic excursion from below the required value to above it or vice versa.

Table 2: Measured factional noise in the flow coefficient during the turbine facility refinement.

\begin{tabular}{|l|c|}
\hline & $(\sigma / \mu)_{1 \mathrm{~s}}$ \\
\hline Before fan PID refinement & $\sim 0.16 \%$ \\
\hline After fan PID, before installation of honeycomb & $0.079 \%$ \\
\hline After fan PID, after installation of honeycomb & $0.050 \%$ \\
\hline
\end{tabular}

This paper is concerned with the development of a computational simulation of the unsteady dynamics of the turbine facility and control system combined. A wide range of parametric studies (adding random noise, varying the control system parameters etc.) were undertaken using the time accurate computational simulation but none reproduced the monotonic excursions. However, when a coupling was introduced between the tangential momentum of the turbine exit flow and the downstream centrifugal fan the computational simulation did exhibit the monotonic excursions. This aerodynamic interaction had been inadvertently introduced into the turbine facility because the direction of rotation of the turbine had been changed during the rebuild. At design the turbine exit swirl is co-rotational with the downstream centrifugal fan and unloads it. Based on the computational simulations a honeycomb was installed between the turbine and the downstream fan. This eliminated the monotonic excursions and the fractional noise of the operating point was further reduced by $37 \%$. When combined with the fan PID control refinement the fractional noise has been reduced by approximately three (Table 2).

There are many publications concerned with the general theory of designing, tuning and testing control systems. There are a few publications, e.g. [8] and [9], which discuss how the shaft speed is controlled in transient facilities. There are also publications, e.g. [10] and [11], which discuss the steady-state control systems, facility configurations and control hardware. The author is not aware of any specific publication concerned with developing and using a computational simulation of a turbine facility combined with its control system to diagnose an unsteady aerodynamic operating problem.

Paper outline: The basic configuration of the turbine facility will be described followed by a summary of the key inputs and outputs of the control system. The main part of this paper will describe the development of the computational simulation for the unsteady dynamics of the turbine and control system combined. This will involve the development of simple lumped parameter models for the unsteady dynamics of the key components within the turbine facility. The solution of the lumped parameter models and the time accurate interaction with the control system will then be described. The computational simulation will then be used to identify the coupling between the turbine exit flow and the centrifugal fan. The unsteady dynamics of the turbine operating point before and after the installation of the honeycomb will be compared followed by a discussion and conclusions.

\section{TURBINE FACILITY}

A schematic of the Peregrine turbine research facility is shown in Fig. 1. The geometrical configuration of the working section is a two-stage low-pressure turbine geometry with representative rotor over-tip and stator under-hub leakages and cavities. The aerodynamic design of the turbine is listed in Table 3. The geometrical arrangement of the working section and the instrumentation are not necessary for this paper but they are described in [5] and [6].

The turbine facility has an atmospheric inlet and, because of the required volumetric flow rate, is operated with the laboratory external doors open. Consequently, the ambient 
conditions, $p_{a m b}$ and $T_{a m b}$ determine the stagnation properties of the flow entering the bellmouth. The static pressure at the exit of the turbine exhaust duct is also $p_{a m b}$. The ambient humidity is used by the control system to determine the appropriate gas constant, $R_{h m d}$ and specific heat capacity at constant pressure, $c_{p, h m d}$ as described in [5].

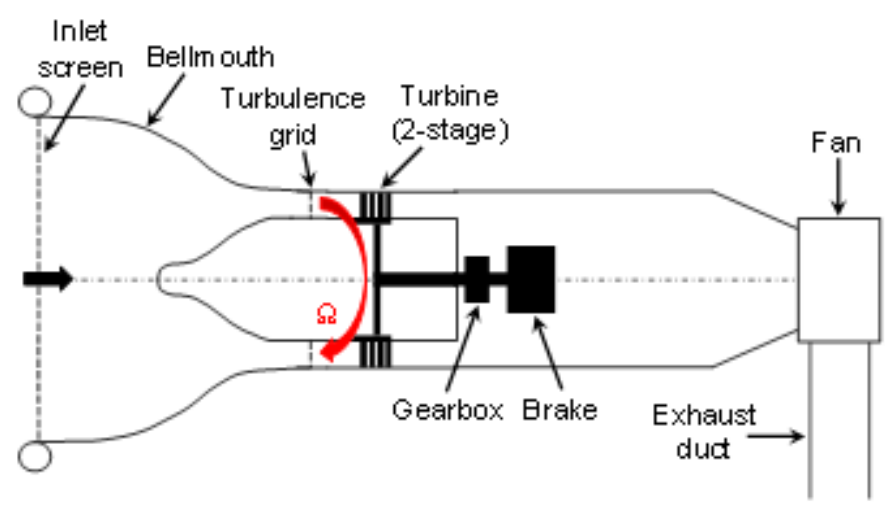

Fig. 1: Schematic of the turbine facility.

Table 3: Design parameters of the LP turbine.

\begin{tabular}{|l|c|}
\hline Number of stages & 2 \\
\hline Mean radius & $0.6477 \mathrm{~m}$ \\
\hline Hub to tip ratio & 0.7 \\
\hline Design flow coefficient (both stages) & 0.822 \\
\hline Design Reynolds number & 148700 \\
\hline Design stage reaction (both stages) & 0.52 \\
\hline Design stage loading (both stages) & 2.02 \\
\hline Nominal blade Mach number & 0.076 \\
\hline Nominal non-dimensional blade speed & 0.0485 \\
\hline Nominal rotational speed & $379 \mathrm{rpm}^{3}$ \\
\hline Nominal volumetric flow rate & $19.7 \mathrm{~m}^{3} \mathrm{~s}^{-1}$ \\
\hline Number of blades: stator, rotor (both stages) & 84,112 \\
\hline Stator exit flow angle (mid-span) & $61.12^{\circ}$ \\
\hline Rotor exit relative flow angle (mid-span) & $-61.75^{\circ}$ \\
\hline Stator axial chord (mid-span) & $50 \mathrm{~mm}$ \\
\hline Rotor axial chord (mid-span) & $35 \mathrm{~mm}$ \\
\hline
\end{tabular}

\section{CONTROL SYSTEM}

Typically an experimental investigation lasts several hours so eliminating the effects of the diurnal ambient variations on the operating point was the motivation for the development of the control system.

There are only two quantities that can be adjusted to obtain the required flow coefficient and Reynolds number: the current, $I_{\text {brake }}$, applied to the eddy-current brake and the speed, $\Omega_{\text {fan }}$, at which the downstream centrifugal fan rotates. In order to automate the control of the facility to track variations in the ambient conditions the Peregrine Control Code was developed by Thomas [7] using LabView. To maintain the required flow coefficient and Reynolds number the full authority control system uses the bellmouth pressure drop, $\Delta p_{\text {bell }}$, the rotational speed of the turbine shaft, $\Omega_{\text {turbine }}$ along with the ambient conditions to determine the required brake torque and fan speed. A simplified schematic of the communication between the turbine facility and the control system is shown in Fig. 2.

The control system also has access to other telemetry. For example, to determine the flow coefficient, which is defined in terms of the area averaged velocity divided by the blade speed, the density at the entry to the turbine working section is needed. This requires the reference stagnation pressure, $p_{t, r e f}$, which is measured by seven Kiel probes upstream of the turbine working section (but downstream of the turbulence grid). By using the reference stagnation pressure, the operation of the turbine facility is independent of any dirt accumulating on either the inlet filter or the turbulence grid.

The control system also checks safety critical quantities and can operate an emergency stop should certain conditions arise.

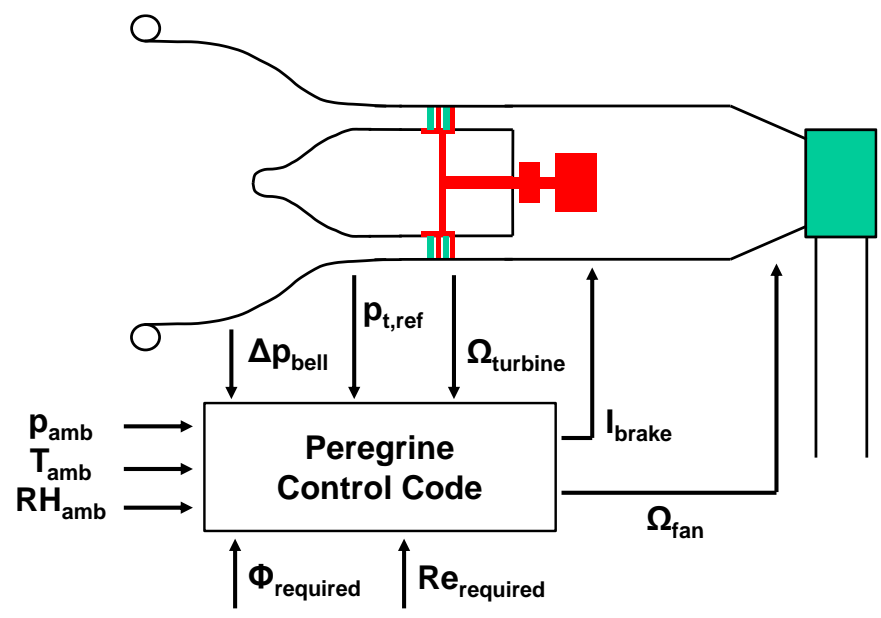

Fig. 2: Key time varying tine quantities exchanged between the turbine facility and the control system (the control system also has access to $p_{t, r e f}$ etc.).

The ambient values provided to the control system are a moving average (over $30 \mathrm{~s}$ ) of the atmospheric conditions upstream of the bellmouth. The time duration for the moving average was empirically determined to eliminate the effects of atmospheric turbulence whilst still allowing the turbine facility to reliably track the diurnal ambient variations.

The key inputs and outputs of the control system can be grouped into three categories depending on whether they are considered to be fixed, slowly-varying during an experiment or time-varying on the timescale of the control system, Table 4.

Table 4: Control system quantities and timescales.

\begin{tabular}{|l|c|}
\hline Category & Timescale \\
\hline Fixed: $\quad \phi_{\text {required }}$ and $R e_{\text {required }}$ & - \\
\hline Slowly-varying: $\quad p_{\text {ambient }}, T_{\text {ambient }}, R H_{\text {ambient }}$ & $\sim 30 \mathrm{~s}$ \\
\hline Time-varying: $\Delta p_{\text {bell }}, \Omega_{\text {turbine }}, I_{\text {brake }}, \Omega_{\text {fan }}$ & $\sim 1 \mathrm{~s}$ \\
\hline
\end{tabular}


The time-varying behaviour of the current applied to the eddy-current brake and the fan speed are determined by a combination of three PID (Proportional, Integral and Differential) controllers, Fig. 3. Two of the controllers, $\operatorname{PID}(\phi)$ and $\operatorname{PID}(R e)$, control the flow coefficient and Reynolds number respectively whilst the third controller, $\mathrm{PID}($ brake), provides a control variable to the eddy-current brake.

The approximate stagnation pressure drop across the turbine is twelve working section dynamic heads $\left(\frac{1}{2} \rho u^{2}\right)$ and the combined stagnation pressure drop through the inlet filter, turbulence grid and all duct work is about nine dynamic heads. Therefore small changes in the flow coefficient by the $\operatorname{PID}(\phi)$ controller do not significantly affect the overall pressure drop through the turbine facility and so only have a small effect on the mass flow rate. Consequently, the mass flow rate is primarily set by the $\operatorname{PID}(R e)$ controller. Thus, the $\operatorname{PID}(\phi)$ and $\mathrm{PID}(R e)$ controllers are effectively orthogonal and the control system updates their control variables every $500 \mathrm{~ms}$.

The eddy-current brake controller, $\operatorname{PID}($ brake) is operated by a sub-system and updates the control variable every $90 \mathrm{~ms}$. This is quicker than the controller for the flow coefficient, $\operatorname{PID}(\phi)$, to enable the brake current to be controlled before the set-point is updated.

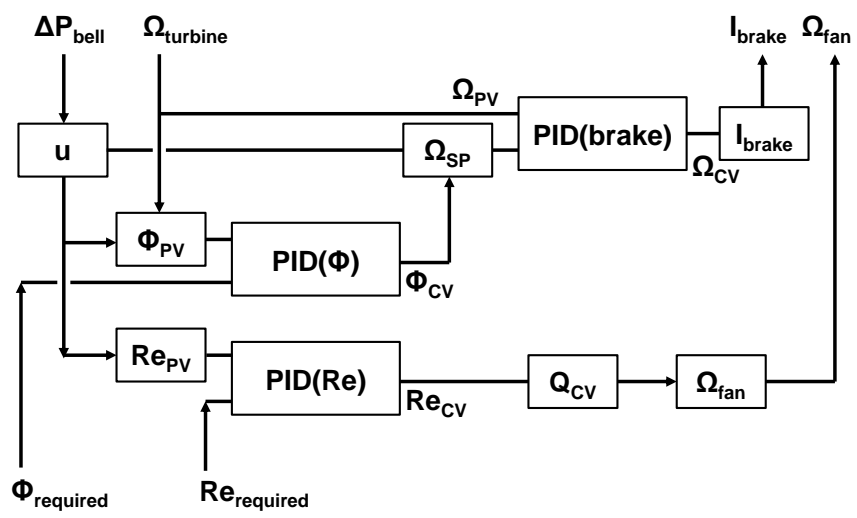

Fig. 3: Control system (simplified) showing the flow of time-varying information between the three PID loops.

\section{UNSTEADY DYNAMIC SIMULATION}

Before describing the simple lumped parameter aerodynamic models for the five key components (bellmouth, turbine, volume, fan and exhaust duct) the five time dependent state variables which are used to represent the instantaneous conditions within the turbine facility will be introduced.

To simulate mass accumulation within the volume between the turbine and the fan the inlet mass flow rate, $\dot{m}_{\text {inlet }}(t)$ (through the bellmouth and turbine) and the exit mass flow rate, $\dot{m}_{\text {exit }}(t)$ (through the fan and exhaust duct) are required. The conditions within the volume are specified by the stored mass, $m_{v o l}(t)$, and the temperature, $T_{v o l}(t)$. The angular velocity of the turbine shaft is specified by $\Omega_{\text {turbine }}(t)$.
Bellmouth dynamics. Although the flow through the bellmouth can be considered incompressible on the timescale at which the control system operates, it is unsteady. Consequently, the relationship between the measured bellmouth pressure drop, $\Delta p_{\text {bell }}$, and the inlet mass flow rate, $\dot{m}_{\text {inlet }}$, must include the effects associated with the acceleration and deceleration of the flow. By considering the bellmouth to be a one-dimensional duct with area $A(x)$, see Fig 4, a lumped parameter model for incompressible unsteady flow through it can be derived.

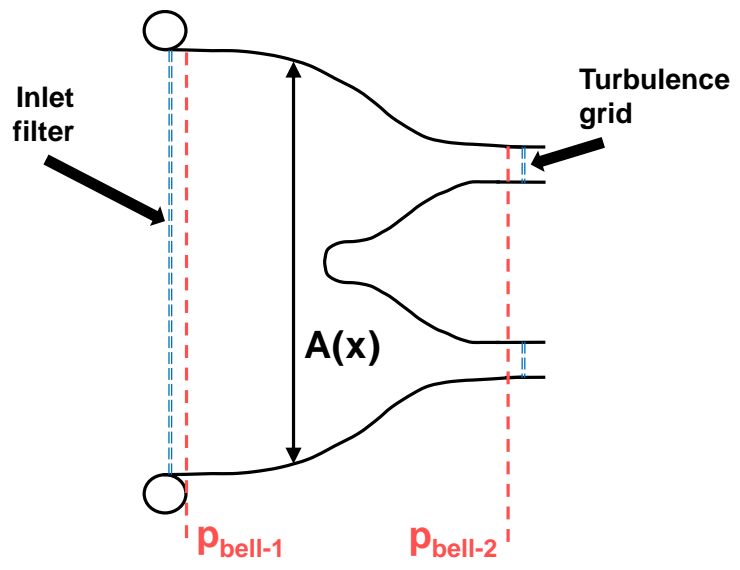

Fig. 4: Schematic of the bellmouth.

Mass conservation for unsteady one dimensional flow through the bellmouth between locations bell-1 (downstream of inlet filter) to bell-2 (upstream of the turbulence grid) can be written:

$$
\frac{\partial}{\partial t}(\rho A)+\frac{\partial}{\partial x}(\rho A u)=0
$$

For incompressible flow, the above can be re-written as:

$$
A(x) u(x, t)=\dot{Q}_{\text {inlet }}(t)
$$

where $\dot{Q}_{\text {inlet }}(t)$ is the volumetric flow rate through the bellmouth. Thus at any axial position within the bellmouth the average velocity can be written as:

$$
u(x, t)=\frac{\dot{Q}_{\text {inlet }}(t)}{A(x)}
$$

The volumetric flow rate through the bellmouth is related to the inlet mass flow rate by:

$$
\dot{Q}_{\text {inlet }}=\frac{\dot{m}_{\text {inlet }}}{\rho_{\text {amb }}} \text { where } \rho_{\text {amb }}=\frac{p_{a m b}}{R_{\text {hmd }} T_{a m b}}
$$

The force-momentum equation for one-dimensional flow through a duct with area $A(x)$ can be written:

$$
\frac{\partial}{\partial t}(\rho A u)+\frac{\partial}{\partial x}\left(\rho A u^{2}+A p\right)=p \frac{\partial A}{\partial x}
$$

Using Eqn. 3 the above can be re-written as:

$$
\frac{1}{A} \frac{\partial}{\partial t}\left(\dot{Q}_{\text {inlet }}\right)+\frac{1}{A} \frac{\partial}{\partial x}\left(\frac{\dot{Q}_{\text {inlet }}^{2}}{A}\right)=-\frac{1}{\rho} \frac{\partial p}{\partial x}
$$


Equation 6 can be integrated to give:

$\frac{\partial}{\partial t}\left(\dot{Q}_{\text {inlet }}\right) \int_{1}^{2} \frac{1}{A} d x+\frac{1}{2} \dot{Q}_{\text {inlet }}^{2}\left[\frac{1}{A_{2}^{2}}-\frac{1}{A_{1}^{2}}\right]=\frac{p_{\text {bell }-1}-p_{\text {bell }-2}}{\rho_{\text {amb }}}=\frac{\Delta p_{\text {bell }}}{\rho_{\text {amb }}}$

Equation 7 is used to determine the rate of change of the volumetric flow rate through the bellmouth given the pressure difference across the bellmouth, $\Delta p_{\text {bell }}$.

In the computational simulation, to determine the static pressure at the entry to the bellmouth ( $p_{\text {bell-1 }}$ which is downstream of the filter) it is necessary to account for the stagnation pressure drop across the inlet filter and the local dynamic head:

$$
p_{\text {bell }-1}=p_{\text {amb }}-\frac{1}{2} \rho_{\text {amb }}\left(\frac{\dot{Q}_{\text {inlet }}}{A_{1}}\right)^{2}\left(1+K_{\text {filter }}\right)
$$

The static pressure at the outlet of the bellmouth $\left(p_{\text {bell }-2}\right.$ which is upstream of the turbulence grid) is related to the reference stagnation pressure, $p_{t, \text { ref }}$, entering the turbine working section:

$$
p_{\text {bell }-2}=p_{t, r e f}-\frac{1}{2} \rho_{\text {amb }}\left(\frac{\dot{Q}_{\text {inlet }}}{A_{2}}\right)^{2}\left(1-K_{\text {grid }}\right)
$$

Because there is approximately a 2.5 dynamic heads stagnation pressure drop across the turbulence grid, the density entering the turbine, $\rho_{\text {ref }}$, is determined by:

$$
\rho_{r e f}=\frac{p_{t, r e f}}{R_{h m d} T_{a m b}}
$$

The flow coefficient at the turbine inlet is given by:

$$
\phi=\frac{u_{\text {turbine-inlet }}}{U} \quad \text { where } \quad u_{\text {turbine-inlet }}=\frac{\dot{m}_{\text {inlet }}}{A_{2} \rho_{\text {ref }}}
$$

Turbine dynamics. The unsteady dynamics of the turbine working section is primarily determined by the shaft inertia and the quasi-steady energy equation.

The torque on the turbine shaft is related to the stagnation enthalpy drop across the turbine, $\Delta h_{t}(\phi)$, by:

$$
\tau_{\text {turbine }} \Omega_{\text {turbine }}=\dot{m}_{\text {inlet }} \Delta h_{t}(\phi)
$$

The stagnation enthalpy drop across the turbine is related to the aerodynamic design, the number of stages, $N_{\text {stage }}$ and the flow coefficient:

$$
\Delta h_{t}(\phi)=N_{\text {stage }}\left(\phi\left(\tan \alpha_{\text {rotor-inlet }}-\tan \alpha_{\text {rotor-exit }}^{\text {rel }}\right)-1\right) U^{2}
$$

The rate of change of the angular velocity of the turbine shaft is determined by:

$$
M_{\text {turbine }} \frac{\partial}{\partial t} \Omega_{\text {turbine }}=\tau_{\text {turbine }}-\tau_{\text {brake }}
$$

where $M_{\text {turbine }}$ is the effective moment inertia of the turbine assembly (shaft, gearbox and eddy-current brake) and $\tau_{\text {brake }}$ is the torque applied by the brake which depends on the current $I_{\text {brake }}$ applied by the control system.

The aerodynamic performance of the turbine is assumed to be quasi-steady, so the stagnation pressure drop across the turbine is given by:

$$
\frac{p_{t, r e f}-p_{t, \text { turbine-exit }}}{\rho_{\text {turbine }}}=\frac{\Delta h_{t}}{\eta_{t t}}
$$

where $\eta_{t t}$ is the total-to-total isentropic efficiency. It is assumed that there is no pressure recovery of the turbine exit flow when it enters the volume, so:

$$
p_{\text {turbine-exit }}=p_{v o l}=\frac{m_{v o l} R T_{v o l}}{V_{v o l}}
$$

where $p_{v o l}$ is the pressure within the volume. The relationship between the stagnation pressure, $p_{t, \text { turbine-exit }}$, and the static pressure, $p_{\text {turbine-exit }}$, at the exit of the turbine is the matching condition which determines the pressures throughout the computational simulation.

Volume dynamics. Mass conservation is applied to the volume between the turbine and the fan to determine the rate of change of the mass within the volume:

$$
\frac{\partial}{\partial t}\left(m_{\text {vol }}\right)=\dot{m}_{\text {inlet }}-\dot{m}_{\text {exit }}
$$

Similarly, energy conservation is used to determine the rate of change of energy within the volume:

$$
\frac{\partial}{\partial t}\left(m_{v o l} c_{v} T_{v o l}\right)=\dot{m}_{\text {inlet }} h_{t, \text { turbine-exit }}-\dot{m}_{\text {exit }} h_{t, v o l}
$$

where $h_{t}$ is the stagnation enthalpy.

Fan dynamics. The fan performance is modelled as quasisteady and is characterised in terms of the volumetric flow rate, $\dot{Q}_{\text {exit }}$, which is defined as follows:

$$
\dot{Q}_{\text {exit }}=\frac{\dot{m}_{\text {exit }}}{\rho_{\text {vol }}} \text { where } \rho_{v o l}=\frac{m_{v o l}}{V_{v o l}}
$$

The pressure rise across the fan is approximated by:

$$
p_{\text {fan-exit }}-p_{v o l}=\left(\frac{\Omega_{\text {fan }}}{\Omega_{\text {fan, nom }}}\right)^{2} D P_{f a n, n o m}\left(\dot{Q}_{\text {exit }} \frac{\Omega_{\text {fan }, n o m}}{\Omega_{\text {fan }}}\right)
$$

where $D P_{\text {fan, nom }}(\dot{Q})$ is the nominal fan performance when operated at the nominal speed $\Omega_{\text {fan,nom }}$. The stagnation pressure at the fan inlet is assumed to be the pressure within the volume.

Exhaust duct dynamics. The equation for the unsteady dynamics of the flow through the exhaust duct can be obtained from those for the bellmouth, Eqn. (7), by assuming a constant area duct. Thus the rate of change of the exit volumetric flow through the exhaust duct is:

$$
\frac{\partial}{\partial t}\left(\dot{Q}_{\text {exit }}\right) \frac{L_{\text {exhaust }}}{A_{\text {exhaust }}}=\frac{p_{\text {fan-exit }}-p_{\text {ambient }}}{\rho_{\text {exit }}}
$$

where $L_{\text {exhaust }}$ and $A_{\text {exhaust }}$ are the length and cross-sectional area of the exhaust duct respectively.

PID controllers: The arrangement of the three PID controllers is shown in Fig. 3 and the PID controller equation for the general quantity $q$ is given by:

$$
q_{C V}=q_{S P}-k_{c}\left\{\left(q_{S P}-q_{P V}\right)+\frac{1}{\tau_{i}} \int\left(q_{S P}-q_{P V}\right) d t-\tau_{d} \frac{d\left(q_{P V}\right)}{d t}\right\} \text { (22) }
$$

where $q_{C V}, q_{S P}$ and $q_{P V}$ are the control-variable, set-point and process-variable respectively and $k_{c}, \tau_{i}$ and $\tau_{d}$ are the PID 
controller constant of proportionality, integration time constant and differential time constant respectively.

Solution method: The time development of the five state variables for the turbine facility $\left(\dot{m}_{\text {inlet }}, \dot{m}_{\text {exit }}, m_{v o l}, T_{v o l}\right.$ and $\Omega_{\text {turbine }}$ ) are governed by five first order differential equations (7, 14, 17, 18 and 21). The five boundary conditions for the turbine facility are:

$$
\begin{gathered}
\text { Bellmouth inlet stagnation pressure }=p_{a m b} \\
\text { Bellmouth inlet stagnation temperature }=T_{a m b} \\
\text { Brake torque }=\tau_{\text {brake }}\left(I_{\text {brake }}\right) \\
\text { Fan speed }=\Omega_{\text {fan }}
\end{gathered}
$$

Exhaust duct exit static pressure $=p_{a m b}$

The values for $I_{b r a k e}$ and $\Omega_{f a n}$ are specified by the time development of the control system where the PID controllers are updated at the appropriate time intervals.

The system of equations is integrated in time using an explicit four-step Runge-Kutta scheme. The global time step is determined by the minimum time-step for each component but is reduced appropriately if it is time to update one of the PID controllers. Because the computational simulation uses simple lumped parameter models for the components, the equations can be quickly integrated for a simulation duration of several hundred seconds.

Turbine exit fan coupling model: At design the exit swirl from the turbine is negative i.e. in the opposite direction to the turbine rotation. So, because the turbine and downstream centrifugal fan rotate in opposite directions, the swirl is in the same direction as the fan rotation. Therefore the turbine exit swirl unloads the centrifugal fan as follows:

$$
\Delta p_{\text {fan,co-swirl }}=\Delta p_{\text {fan,no-swirl }}+\rho_{v o l} \Omega_{\text {fan }}(r v)_{\text {turbine-exit }}\left(t_{p r v}\right)
$$

Where $(r v)_{\text {turbine-exit }}$ is the radius multiplied by the tangential velocity of the flow leaving the turbine (a negative quantity and $\Omega_{\text {fan }}$ is positive). The value of $(r v)_{\text {fan-inlet }}(t)$ is assumed to correspond to $(r v)_{\text {turbine-exit }}\left(t_{p r v}\right)$ the value at the turbine exit at the previous time, $t_{p r v}$, at which the fluid entering the fan left the turbine. The previous-time, $t_{p r v}$, is determined by:

$$
\int_{t_{p r v}}^{t} \dot{Q}_{\text {inlet }}(t) d t=V_{v o l}
$$

which is when the integral of the volumetric flow rate through the turbine equals the volume, $V_{v o l}$, between the turbine exit and the fan inlet.

\section{FACILITY DYNAMICS WITHOUT HONEYCOMB}

The measured fractional variation in the flow coefficient over a period of $400 \mathrm{~s}$ is shown in Fig. 5. The corresponding fractional noise measured in any one second interval, $(\sigma / \mu)_{1 \mathrm{~s}}$ is $0.079 \%$, see Table 2. Whilst this is small, there are occasions, e.g. at time $260 \mathrm{~s}$, where there is a monotonic excursion from above to below the required value during an interval of approximately $25 \mathrm{~s}$. This suggests that whilst the control system is working as desired, there are instances where it is "losing control" of the facility.

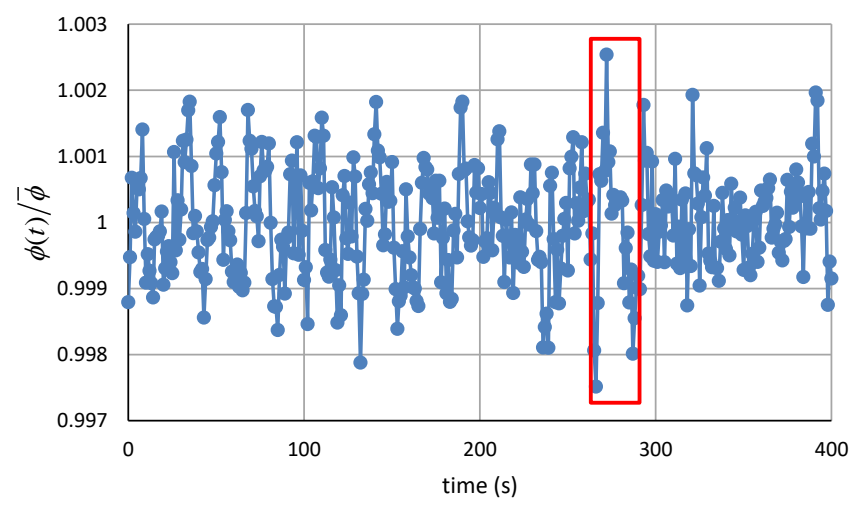

Fig. 5: Measured fractional variation of flow coefficient prior to installation of honeycomb upstream of the fan. The red box indicates the monotonic excursion.

To determine the characteristic response time of the turbine and control system combined, autocorrelations of the time variation of the Reynolds number and flow coefficient are shown in Fig. 6. The autocorrelation is non-dimensionalised by the standard deviation so it has unit value at zero time delay. A convenient measure of the response time of the turbine facility and control system combined will be taken to be the autocorrelation time delay corresponding to the first zero crossing. Prior to the installation of the honeycomb the time delays are $3 \mathrm{~s}$ and $4 \mathrm{~s}$ for the Reynolds number and flow coefficient respectively.

An overall characteristic time of 3 to $4 \mathrm{~s}$ is consistent with the fan and brake controller update intervals of $500 \mathrm{~ms}$ and $90 \mathrm{~ms}$ respectively. However, it is not consistent with a monotonic excursion from above to below the required operating value lasting for approximately $25 \mathrm{~s}$.

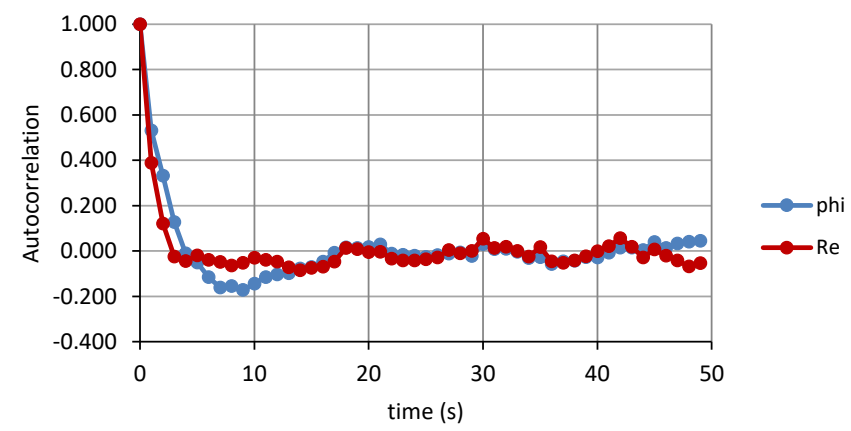

Fig. 6: Autocorrelation of the flow coefficient and Reynolds number prior to installation of the honeycomb upstream of the fan. 


\section{COMPUTATIONAL SIMULATION INVESTIGATION}

A wide range of studies were investigated using the computational simulation to try and reproduce the monotonic excursions shown in Fig. 5. These included: the addition of random Gaussian gusts to the ambient conditions; varying the constants within the PID controllers; adding random Gaussian noise to the telemetry and control quantities. It was not until the aerodynamic coupling of the moment of momentum of the fluid passing between the turbine exit and fan inlet was incorporated (Eqns 24, 25) in the computational simulation was it possible to reproduce the monotonic excursions. Figure 7 shows such a computational simulation where the monotonic excursion is clearly calculated at approximately $320 \mathrm{~s}$.

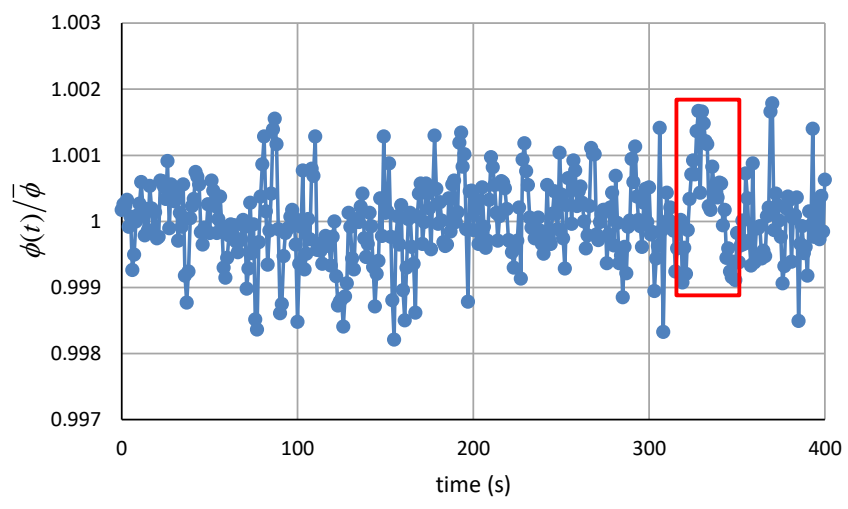

Fig. 7: Calculated fractional variation of flow coefficient with turbine-fan coupling model included. The red box indicates the monotonic excursion.

\section{FACILITY DYNAMICS WITH FAN INLET HONEYCOMB}

Based on the investigations using the computational simulation, a honeycomb was installed into the turbine facility upstream of the fan to eliminate the aerodynamic coupling between the turbine exit swirl and fan. The measured fractional variation of the flow coefficient after the installation of the honeycomb is shown in Fig. 8. The fractional noise has been reduced to $0.050 \%$, Table 2, which is a $37 \%$ reduction compared to the value without the honeycomb. There are no lengthy excursions from above to below the required value (but there are one-sided excursions).

To determine the characteristic time-scale of the combined turbine facility and control system after the installation of the honeycomb, the autocorrelations of the Reynolds number and flow coefficient are shown in Fig. 9. Both the Reynolds number and flow coefficient characteristics times are approximately $4 \mathrm{~s}$ suggesting that both aspects of the turbine facility dynamics are similarly controlled. The negative portion of the autocorrelation of the flow coefficient which occurs at about a time delay of eight seconds has a smaller magnitude once the honeycomb has been installed, Fig. 9, compared to without the honeycomb, Fig. 6. This suggests that a periodic oscillation in the flow coefficient within the turbine facility has been reduced.

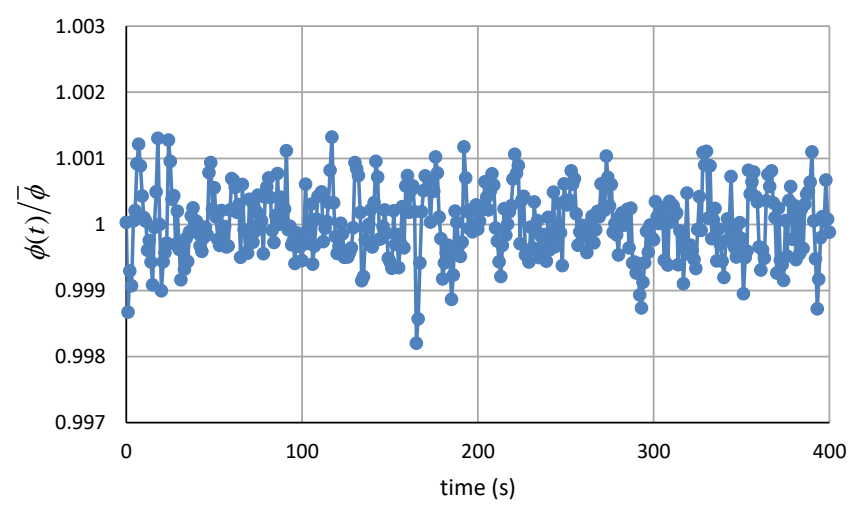

Fig. 8: Measured fractional variation of flow coefficient after installation of honeycomb upstream of the fan.

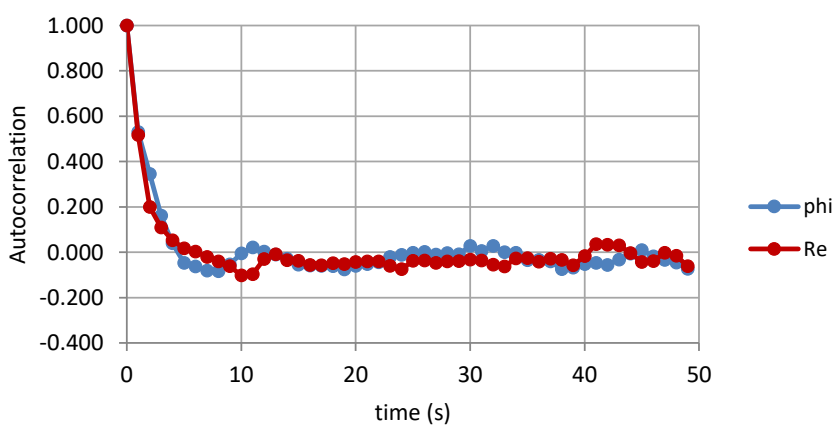

Fig. 9: Autocorrelation of the flow coefficient and Reynolds number after installation of the honeycomb upstream of fan.

\section{DISCUSSION}

The development of a real-time full authority computer control system for a turbine facility is a major undertaking and it can be difficult to refine the control system without risking the integrity of the facility. This paper has described the development of a time accurate computational simulation of the unsteady dynamics of the turbine and control system combined.

The computational simulation has been used to identify the aerodynamic interaction between the turbine exit flow and the centrifugal fan which was responsible for the control system occasionally "losing-control" of the facility. The addition of the honeycomb between the turbine exit and the downstream fan reduced the fractional noise in any one second interval by $37 \%$. Because the addition of the honeycomb prevented any bulk swirl in the turbine exit flow unloading the centrifugal fan, the maximum Reynolds number that the facility can achieve increased by about $3 \%$ which corresponds to about a $10 \%$ increase in turbine shaft power output.

Excluding the monotonic excursion, the scatter in the calculated fractional variation of the flow coefficient shown in Fig. 7 is due to the random Gaussian noise added into the computational simulation. The scatter (excluding monotonic excursion) in the measured fractional variation of the flow 
coefficient before and after the installation of the honeycomb, Figs 5 and 8 respectively, are similar to that produced by the computational simulation. This suggests that the measured scatter may be due to random noise. The autocorrelations, shown in Figs 6 and 9, of the measured data show that for time delays greater than $4 \mathrm{~s}$ the experimentally observed scatter is uncorrelated - i.e. independent.

For data which contains independent random noise with standard deviation, $\sigma$, averaging over $\mathrm{N}$ measurements reduces the standard deviation to $\sigma / \sqrt{N}$ (standard error of the mean). The fractional noise measured in any one second interval, $(\sigma / \mu)_{1 \mathrm{~s}}$, after the fan PID and honeycomb refinements is $0.050 \%$ (Table 2). Because the noise is uncorrelated for time delays greater than $4 \mathrm{~s}$, averaging repeat measurements during steady-state operation reduces the noise. For example, averaging 120 one-second samples yields a fractional noise, $(\sigma / \mu)_{120 \mathrm{~s}}$, of $0.004 \%$ (Table 1 ). This is one advantage of using a nominally steady-state turbine facility. It also highlights the benefit of a refined control system which achieves a low value of the factional noise (before the refinement it would have taken over a thousand samples to get such consistency).

This paper has been concerned with low-speed turbine research facilities with atmospheric inlets. However, the lumped parameter unsteady dynamic model can be extended to high-speed facilities (where the inlet stagnation temperature must also be controlled) and closed circuit facilities (where cooling is usually required to counteract the temperature rise during the compression process).

It is worth noting that a low-speed compressor facility, where the operating point is controlled by specifying the rotational speed and the area of a downstream throttle, the direct aerodynamic coupling between the compressor and the throttle ensures that a change in the rotational speed only affects the Reynolds number. Further, above a critical value, compressor performance is a weak function of Reynolds number so any small change in the rotational speed has little effect on the measurements. Consequently, once set, a lowspeed compressor facility will remain at the required flow coefficient and close to the required Reynolds number.

\section{CONCLUSIONS}

During re-commissioning of the Peregrine turbine facility, a refinement to the PID loop control of the fan speed reduced the factional noise in the operating point by approximately $50 \%$. It was then observed that there were monotonic excursions in the operating point on a timescale longer than that of the control system. The investigation of this behaviour motivated the development of the time accurate computational simulation for the turbine facility and control system combined.

1) Lumped parameter models have been obtained for all the key components within the turbine facility and implemented within a time accurate computational simulation which also includes the temporal behaviour of the control system.

2) Investigations of the turbine facility and control system combined using the computational simulation identified an aerodynamic coupling between the turbine exit flow and the downstream fan. This aerodynamic coupling had been introduced in the rebuilt configuration of the Peregrine facility because the rotation direction of the turbine had been changed.

3) The addition of a honeycomb between the turbine exit and the fan inlet reduced the fractional noise in the turbine facility by approximately $37 \%$. There was also a $3 \%$ increase in the maximum Reynolds number that could be achieved within the turbine facility (about $10 \%$ power).

4) Overall, the refinement to the fan PID loop and the addition of the honeycomb reduced the fractional noise in the turbine facility by approximately a factor of three. This corresponds to approximately a nine fold reduction in the number of repeated measurements necessary to achieve a given level of data consistency.

\section{NOMENCLATURE}

$A=$ cross sectional area

$c_{p, h m d}=$ humid air specific heat capacity at constant pressure

$D P=$ nominal fan performance

$h_{t}=$ stagnation enthalpy

$\eta_{t t}=$ isentropic total-to-total efficiency

$I_{\text {brake }}=$ control current applied to turbine brake

$k_{c}=$ PID proportional control constant

$\mathrm{K}=$ stagnation pressure loss coefficient

$L=$ duct length

$m, \dot{m}=$ mass, mass flow rate

$M=$ moment of inertia

$N=$ number of data points, number of stages

$\dot{Q}=$ volumetric flow

$p, p_{t}=$ static, stagnation pressure

PID = Proportional, Integral, Differential controller

$(r v)=$ moment of tangential velocity

$R_{\text {hmd }}=$ humid air gas constant

$R e=$ Reynolds number

$R H=$ Relative humidity

$t, t_{p r v}=$ time, previous time (in Eqns 24 and 25)

$T, T_{t}=$ static, stagnation temperature

$\rho=$ density

$\sigma=$ standard deviation

$u, v=$ axial, tangential velocity

$U=$ blade speed

$\mu=$ mean value

$\tau=$ torque

$\tau_{i}, \tau_{d}=$ PID integral and differential time delay

$\phi, \bar{\phi}=$ flow coefficient, mean flow coefficient

$\Omega=$ angular velocity

$V=$ volume

$\mathrm{x}=$ axial position 


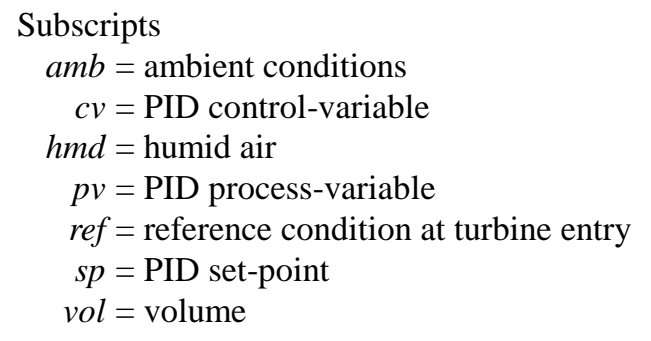

\section{ACKNOWLEDGMENTS}

The Author wishes to thank Rolls-Royce, in conjunction with the ATI, for supporting the upgrade of the Peregrine facility through the SAMULET programme and for the permission to publish. The contribution of Dr K.R. Evans in building and operating the facility is gratefully acknowledged. The Author is also grateful for the information provided by Prof. P.R.N. Childs and the draft paper from Mr J. Eitenmuller.

\section{REFERENCES}

1 Dunkley, M.J., 1998, “The Aerodynamics of Intermediate Pressure Turbines", PhD Thesis, Cambridge University.

2 Beard, P.F., Smith, A.D. and Povey, T., 2014, "Effect of Combustor Swirl on Transonic High Pressure Turbine Efficiency", ASME J. Turbomach, 136(1): 011002-01100212. doi:10.1115/1.4024841.

3 Kirollos, B., Lubbock, R., Beard, P.F., Goenaga, F., Rawlinson, A., Janke, E. and Povey, T., 2017, "ECAT: An Engine Component Aerothermal Facility at the University of Oxford", ASME Paper No. GT2017-64736, pp. V02AT40A032; 14 pages. doi:10.1115/GT2017-64736.
4 Eitenmuller, J., Schiffer, H-P., Wihelm, M., Gresser, L., Ostrowski, T., Leichtfuss, S., Lyko, C., and Nail, S., 2019, "Highly Accurate Delta Efficiency Measurements at the Large Scale Turbine Rig," ASME GT2019-90294 (draft).

5 Evans, K. R., and Longley, J. P., 2017, “Accounting for Uncontrolled Variations in Low-Speed Turbine Experiments," ASME J. Turbomach, 139(10): 101005101005-12. doi: 10.1115/1.4036342.

6 Evans, K. R., and Longley, J. P., 2017, "Clocking in LowPressure Turbines", ASME J. Turbomach.; 139(10): 101003-101003-15. doi: 10.1115/1.4036341

7 Thomas, R., Peregrine Control code, 2010.

8 Goodisman, M.I., Oldfield, M.L.G., Kingcombe, R.C., Jones, T.V., Ainsworth, R.W., and Brooks, A.J. 1992, “An Axial Turbobrake," ASME J. Turbomach. 114(2): 419-425. doi: 10.1115/1.2929160.

9 Epstein, A.H., Guenette, G.R., and Norton, R.J.G., 1984, "The MIT Blowdown Turbine facility," Proc. ASME. 79498; Volume 4: Heat Transfer; Electric Power, V004T09A011, 84-GT-116, doi: 10.1115/84-GT-116.

10 Turner, A.B., Davies, S.J., Childs, P.R.N., Harvey, C.G., and Millward, J.A., 2000, "Development of a Novel Gas Turbine Driven Centrifugal Compressor," Proceedings of the Institution of Mechanical Engineers, Part A, Journal of Power and Energy, Vol. 214, Part A, pp. 423-437, 2000.

11 Sell, M., Schlienger, J., Pfau, A., Treiber, M., Abhari, R.S., 2001, "The 2-stage Axial Turbine Tests Facility 'LISA',"

Proc. ASME Paper 2001-GT-0492, pp. V001T03A076; 9 pages, doi: 10.1115/2001-GT-0492. 